\title{
Successful endoscopic treatment of walled-off pancreatic necrosis complicated with pancreaticopleural and pancreaticocolonic fistulas
}

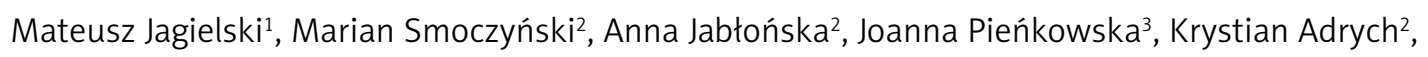
Marek Jackowski ${ }^{1}$

\author{
'Department of General, Gastroenterological and Oncological Surgery, \\ Collegium Medicum, Nicolaus Copernicus University, Torun, Poland \\ ${ }^{2}$ Department of Gastroenterology and Hepatology, Medical University of Gdansk, \\ Gdansk, Poland \\ ${ }^{3}$ Department of Radiology, Medical University of Gdansk, Gdansk, Poland
}

Submitted: 20 April 2017

Accepted: 22 September 2017

Arch Med Sci 2020; 16 (2): 471-474

DOI: https://doi.org/10.5114/aoms.2017.70658

Copyright @ 2020 Termedia \& Banach

A 53-year-old man was admitted to a different medical center due to abdominal pain with nausea in December 2015. Severe acute pancreatitis of alcoholic etiology was recognized. Conservative treatment was applied. Acute necrotizing pancreatitis was discovered in imaging examinations (Figure $1 \mathrm{~A}$ ). The computed tomography severity index (CTSI) showed 8 points. Gradual liquidation and encapsulation of necrosis of the pancreatic tail and peripancreatic tissues (with no features of infection) were confirmed in subsequent imaging examinations, which were done in the next days of hospitalization. The patient was released home after 3 weeks of hospitalization.

Later on the same patient was admitted to our department in January 2016 with a 1-week history of abdominal pain, fever, dyspnea and diarrhea. The laboratory blood tests done after admission revealed increased parameters of inflammation (CRP $263 \mathrm{mg} /$; leukocytosis $22 \mathrm{G} / \mathrm{l}$ ). Blood cultures were negative. Contrast-enhanced computed tomography (CECT) showed walled-off pancreatic necrosis (WOPN) and left sided pleural effusion (Figure 1 B). The infected WOPN was recognized in the described case. Empirical antibiotic therapy (tazobactam with piperacillin) was applied on the first day of hospitalization. The patient was qualified for endoscopic treatment of WOPN on the basis of clinical symptoms related to the presence of necrotic collection and the results of CECT of the abdomen. Endoscopic transmural drainage was not possible (the distance between the gastrointestinal tract and WOPN exceeded $15 \mathrm{~mm}$ in endoscopic ultrasonography imaging); therefore active transpapillary drainage was started via the major duodenal papilla (Figure $1 \mathrm{C}$ ). A fluid sample from the collection was taken for microbial culture during the endoscopic procedure. Positive culture of the collection's content confirmed the presence of Enterococcus faecalis, Enterococcus faecium and Escherichia coli. The antibiotic therapy, applied previously, was continued for the next 44 days in accordance with culture of the collection's content. Therapeutic thoracentesis was also performed. Laboratory analysis of the pleural fluid showed increased concentration of amylase $(2346 \mathrm{U} / \mathrm{l})$ and low protein concentration (below $3 \mathrm{~g} / \mathrm{dl}$ ). After 7 days of treatment contrast injected via a nasal drain filled the necrotic collection with con-

\author{
Corresponding author: \\ Mateusz Jagielski PhD \\ Department of \\ Gastroenterology \\ and Hepatology \\ Medical University \\ of Gdansk \\ 17 Smoluchowskiego St \\ 80-214 Gdansk, Poland \\ Phone: +48 583493640 \\ Fax: +48583493650 \\ E-mail: matjagiel@gmail.com
}



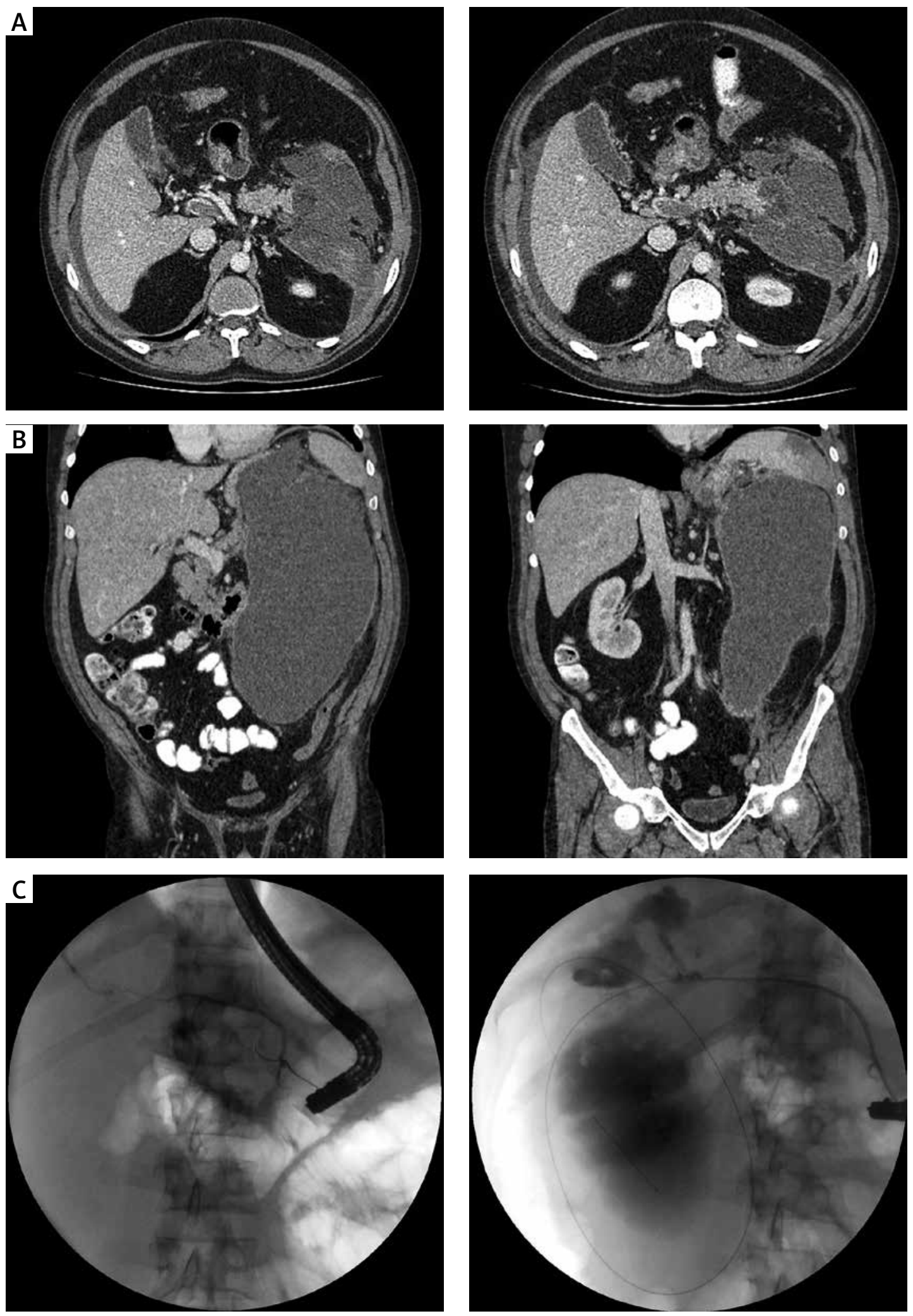

Figure 1. A - Contrast-enhanced computed tomography (CECT) of the abdomen done on tenth day of acute necrotizing pancreatitis. The examination revealed features of necrosis of the pancreatic tail and peripancreatic tissues. B - Abdominal computed tomography before the drainage showed walled-off pancreatic necrosis sized $300 \times 250$ $\times 180 \mathrm{~mm}$, multiple foci of splenic infarction and fluid in the left pleural cavity. C - Endoscopic retrograde pancreatography showing complete rupture of the main pancreatic duct in the region of the pancreatic tail. The guidewire introduced into the main pancreatic duct formed a loop in the cavity of the necrotic collection as it passed through the rupture site 

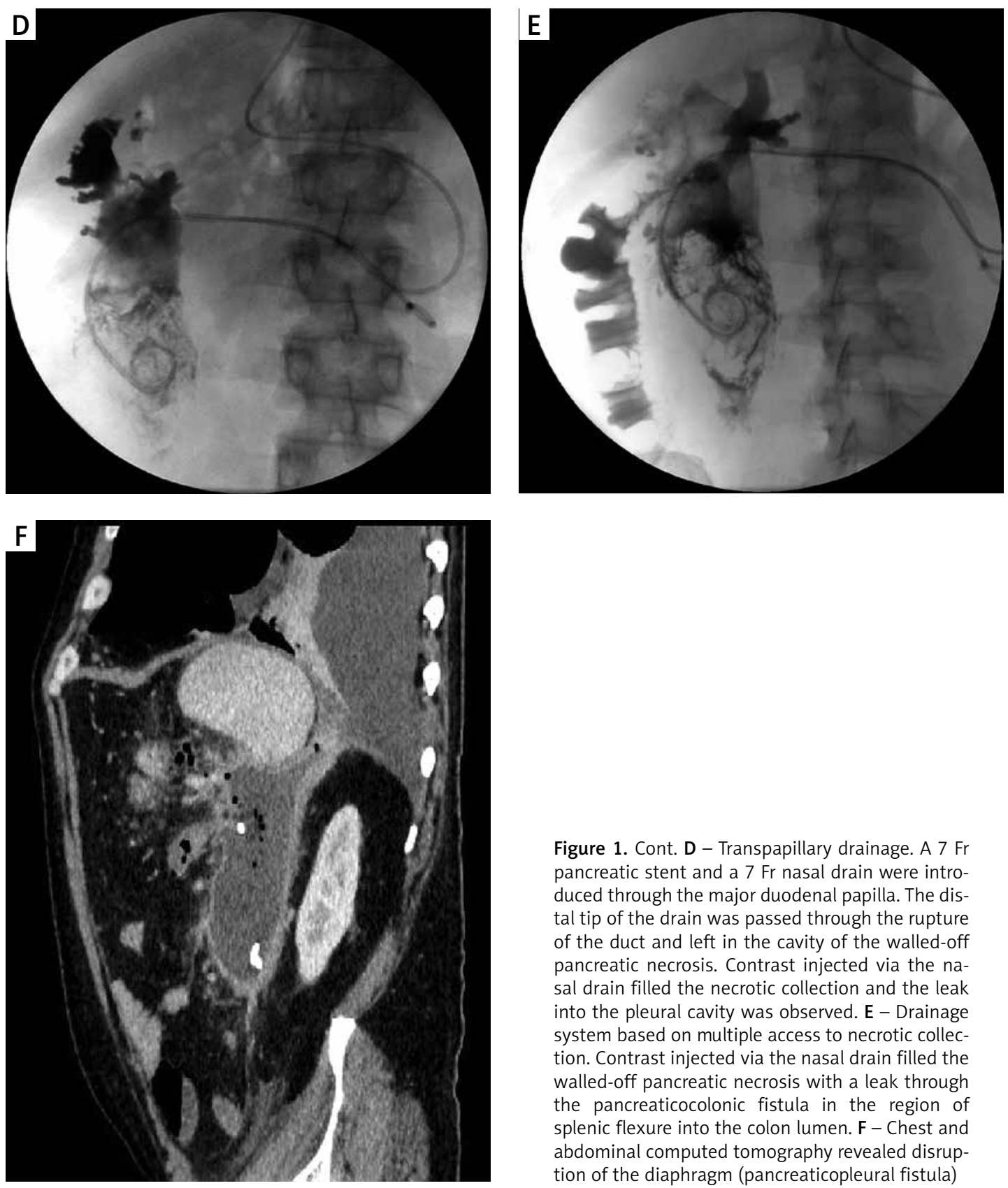

Figure 1. Cont. D - Transpapillary drainage. A $7 \mathrm{Fr}$ pancreatic stent and a $7 \mathrm{Fr}$ nasal drain were introduced through the major duodenal papilla. The distal tip of the drain was passed through the rupture of the duct and left in the cavity of the walled-off pancreatic necrosis. Contrast injected via the nasal drain filled the necrotic collection and the leak into the pleural cavity was observed. E - Drainage system based on multiple access to necrotic collection. Contrast injected via the nasal drain filled the walled-off pancreatic necrosis with a leak through the pancreaticocolonic fistula in the region of splenic flexure into the colon lumen. $\mathrm{F}$ - Chest and abdominal computed tomography revealed disruption of the diaphragm (pancreaticopleural fistula)

trast leak into the left pleural cavity (Figure $1 \mathrm{D}$ ) and colon (Figure $1 \mathrm{E}$ ). CECT showed disruption of the diaphragm (Figure $1 \mathrm{~F}$ ) and pancreaticocolonic fistula. After another three weeks of transpapillary drainage partial regression of WOPN was observed with no leak of contrast outside the necrotic collection. After 43 days of active drainage the regression of WOPN and resolution of symptoms were confirmed (Figure $1 \mathrm{G}$ ). The nasal drain was removed and the pancreatic stent was exchanged.

The complete regression of collection and no presence of pancreaticopleural and pancreaticocolonic were confirmed in follow-up CECT of the abdomen during the next hospitalization, which took place 3 months after the end of drainage.

During the endoscopic procedure not only was the pancreatic stent removed, but also no disruption of the main pancreatic duct was noted. After 1-year follow-up the patient is found to be in good general condition without clinical symptoms. Recurrence of collection and pancreatic fistulas was not observed in follow-up imaging examinations.

Pancreaticopleural fistulas are considered to be rare complications of chronic or acute pancreatitis. Endotherapy is an effective treatment of pancreaticopleural fistulas [1, 2]. Pancreaticocolonic fistulas are much more serious complications of acute pancreatitis associated with high morbidity [3-5] due to accompanying septic complications and hemorrhage. For pancreaticocolonic fistulas 

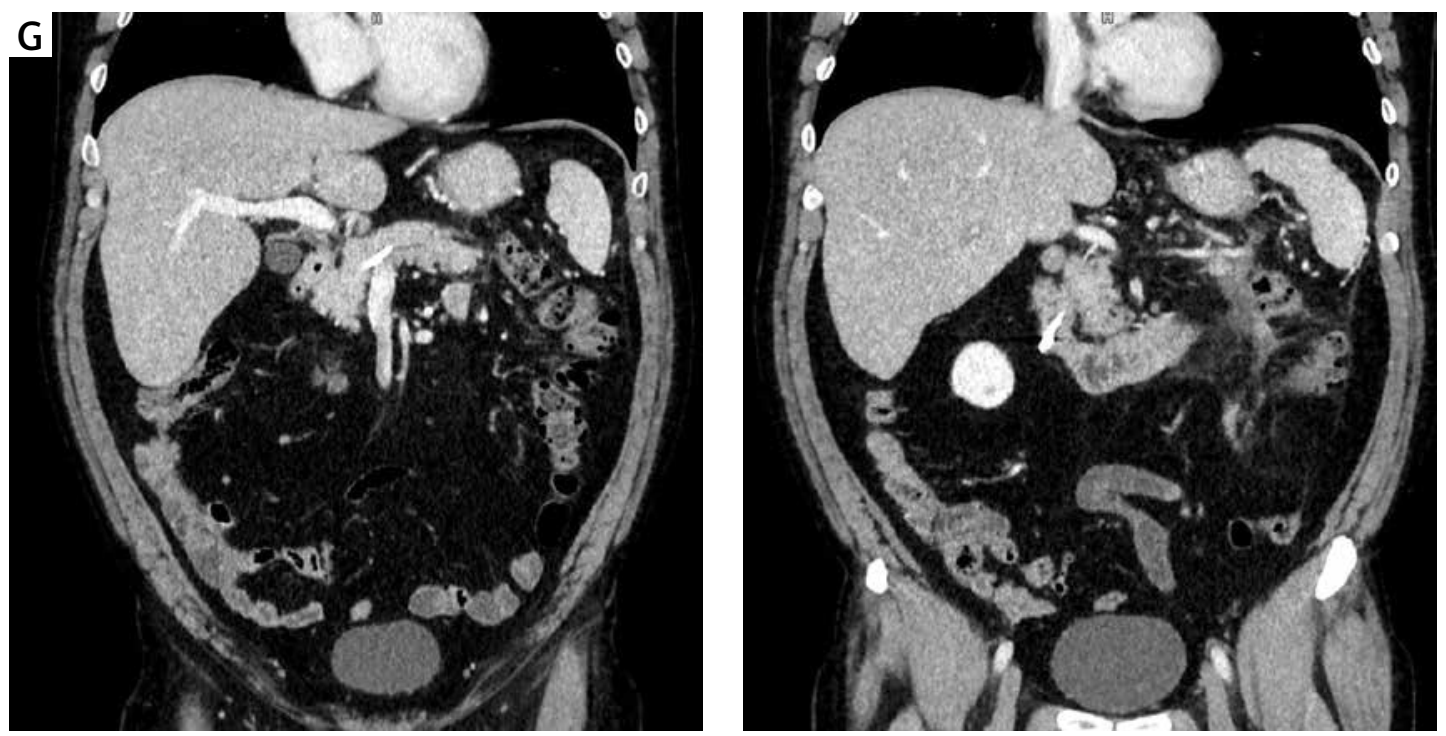

Figure 1. Cont. G - Control abdominal computed tomography after endoscopic treatment showed complete regression of walled-off pancreatic necrosis. Previously observed pancreatic fistulas were no longer seen

surgery is the treatment of choice [3], particularly in the case of septic complications. Conservative treatment of pancreaticocolonic fistulas can lead to their spontaneous closure and is reserved for stable patients $[3,6]$. In the present case pancreaticocolonic and pancreaticopleural fistulas were the results of spontaneous fistulization of WOPN into the lumen of the colon and pleural cavity in the late phase of acute necrotizing pancreatitis, which may explain the quite good clinical condition of the patient.

Transpapillary drainage as the only access to the WOPN is still a controversial and uncommonly reported method of treatment [7]. Law et al. described endoscopic necrosectomy through the fistula between the WOPN and the colon [8]. In the present case colonic fistula provided an additional access to the WOPN that enabled the flow of necrotic content into the colon during active drainage.

In conclusion, this report describes the first case in the world of successful endoscopic treatment of walled-off pancreatic necrosis complicated with pancreaticopleural and pancreaticocolonic fistulas.

\section{Conflict of interest}

The authors declare no conflict of interest.

\section{References}

1. Koshitani T, Uehara Y, Yasu T, et al. Endoscopic management of pancreaticopleural fistulas: a report of three patients. Endoscopy 2006; 38: 749-51.

2. Dhebri AR, Ferran N. Nonsurgical management of pancreaticopleural fistula. JOP 2005; 6: 152-61.

3. Mohamed SR, Siriwardena AK. Understanding the colonic complications of pancreatitis. Pancreatology 2008; 8: 153-8.
4. Rerknimitr R, Lakananurak N, Prueksapanich P, et al. A fatal case of a colonic fistula communicating with a walled-off area of pancreatic necrosis. Endoscopy 2014; 46 Suppl 1 UCTN: E30-31.

5. Tsiotos GG, Smith CD, Sarr MG. Incidence and management of pancreatic and enteric fistulas after surgical management of severe necrotizing pancreatitis. Arch Surg 1995; 130: 48-52.

6. Green BT, Mitchell RM, Branch MS. Spontaneous resolution of a pancreatic-colonic fistula after acute pancreatitis. Am J Gastroenterol 2003; 98: 2809-10.

7. Smoczyński M, Jagielski M, Jabłońska A, Adrych K. Transpapillary drainage of walled-off pancreatic necrosis a single center experience. Videosurgery Miniinv 2015; 10: 527-33.

8. Law R, Wong Kee Song LM, Baron TH. Simultaneous transgastric and transcolonic debridement of walledoff pancreatic necrosis. Gastrointest Endosc 2014; 80: 1172. 